\title{
Presentación del monográfico sobre ciencia recreativa
}

\author{
Rafael García-Molina \\ Editor Adjunto de la Revista Eureka sobre Enseñanza y Divulgación de las Ciencias. Coordinador de este \\ número extraordinario dedicado a la ciencia recreativa
}

Departamento de Fisica, Centro de Investigación en Óptica y Nanofísica. Universidad de Murcia. Murcia. España.rgm@um.es

Foreword to the special issue devoted to recreational science

A short overview of the section devoted to recreational science is presented, together with a brief description of each one of the papers that are published in this issue.

Keywords: Recreational science; Physics; Chemistry; Biology; Geology; Astronomy.

La sección de ciencia recreativa apareció en la Revista Eureka sobre Enseñanza y Divulgación de las Ciencias (REurEDC, a partir de ahora) por primera vez en abril de 2007, en el segundo número del cuarto volumen. En las normas de publicación de esta sección se hacía constar su propósito: «...presentar experiencias científicas u otras actividades que resulten amenas (y, en ocasiones, sorprendentes) a la vez que formativas, y que puedan emplearse por los docentes para estimular el interés por la ciencia y suscitar preguntas que ayuden a comprender los fenómenos y conceptos científicos.», poniendo énfasis en la sencillez de la presentación y la disponibilidad de los materiales: «Se valorará positivamente la simplicidad de diseño, la sencillez de la construcción y presentación, así como el uso de materiales sencillos y asequibles».

El acierto en la decisión que se tomó hace ahora casi un lustro se puede constatar en la calidad de los 32 artículos publicados hasta la fecha, lo cual resulta en una media de aproximadamente dos artículos en cada ejemplar de la revista.

El uso de experiencias de ciencia recreativa en el ámbito docente contribuye, sin duda, a mejorar la actitud de los estudiantes hacia las disciplinas científicas (Solbes et al. 2009). Por ello, actualmente es frecuente que este tipo de actividades se incorporen a la práctica docente, junto con (y no como alternativa a) otras propuestas didácticas. No obstante, como en toda actividad que se lleve al aula, es necesario efectuar estudios adicionales que analicen la efectividad de estos recursos didácticos en el proceso de enseñanza-aprendizaje. En este sentido se publicó una oportuna reflexión en el editorial de REurEDC donde se requería la realización de trabajos que valoren la fundamentación y contextualización de este tipo de propuestas (Oliva 2008).

El origen de este monográfico sobre ciencia recreativa se remonta a septiembre de 2009, cuando comenzó a rondar por mi cabeza la posibilidad de recopilar una selección de trabajos que recogieran la variedad de experiencias, contextos y profesorado involucrados en estas actividades. Poco tiempo después, el Comité Editorial de REurEDC consideró la posibilidad de preparar un número monográfico sobre ciencia recreativa, ${ }^{1}$ en sintonía con la línea editorial recién iniciada por REurEDC de añadir a los tres números anuales uno extra dedicado monográficamente a un tema.

\footnotetext{
${ }^{1}$ ¿Casualidad o telepatía? En todo caso, queda constancia de la empatía que nos une en esta aventura de contribuir a mejorar la enseñanza de las ciencias.
} 
Inmediatamente se enviaron las cartas recabando colaboraciones para participar en este proyecto. Los destinatarios de las invitaciones eran personas cuya trayectoria en el campo de la divulgación científica estaba acreditada por su participación en certámenes, talleres, cursos, etc. donde se transmitía al público la pasión por la ciencia mediante la realización de experimentos, sencillos o espectaculares, que contagiaban a los espectadores las ganas de conocer más sobre las leyes, los conceptos o los fenómenos científicos.

En menos de un mes comenzaron a llegar las respuestas, la mayoría aceptando embarcarse en este proyecto. Algunas personas declinaron la invitación por falta de tiempo y otras, sencillamente, no respondieron.

Los artículos recibidos fueron revisados siguiendo los criterios de calidad que venimos aplicando en REurEDC: valor intrínseco del trabajo, interés para los lectores de la revista, redacción y presentación adecuadas... Aparte de la propia revisión efectuada por el comité editorial, cada trabajo se remitió a dos evaluadores externos a la revista. Tan solo cuatro trabajos fueron rechazados o retirados por sus autores, mientras que veinte se aceptaron tras los típicos intercambios de correos entre evaluadores, autores y editores. Aunque todo este proceso ha durado casi un año, estoy convencido que el resultado ha merecido la pena. Por ello quiero agradecer, tanto a los autores como a los evaluadores, el excelente trabajo que han efectuado.

El elenco de colaboraciones no pretende ser exhaustivo en cuanto a los autores y los temas que aparecen en el monográfico. Pero sí que es una excelente muestra del tipo de actividades que puede desarrollarse, tanto en el aula como en cualquier tipo de certamen o feria de la ciencia, para estimular el interés de la sociedad por la ciencia y, por supuesto, para mejorar la praxis docente.

Este monográfico consta de 20 artículos (además de esta presentación). En el primero se realiza una incursión histórica por la ciencia recreativa, se destacan sus potencialidades didácticas y se apuesta firmemente por su incorporación a la práctica docente. Los 19 restantes se han organizado agrupándolos en áreas científicas tradicionales: Física (7), Química (6), Biología (2), Geología (2) y Astronomía (2).

El primer artículo dedicado a la Física muestra cómo unos simples tubos de PVC sirven para hacer música y también para medir la velocidad del sonido en el aire, tal como explican Ana Cros y Chantal Ferrer Roca en el trabajo «Física por un tubo. Mide la velocidad del sonido en el aire y diviértete con los tubos sonoros», cuyo sugestivo título es un claro guiño de complicidad con el lector.

Julio Güémez muestra que un juguete -o artículo de regalo-, tal como el pajarito bebedor, puede dar lugar a una profunda y amena clase sobre termodinámica. El título del artículo es explícito «La física del pájaro bebedor» y me trae a la memoria una polémica sobre la enseñanza de la física que tuvo lugar hace casi una década (Lozano Leyva 2002, Romero Peinado 2002, Nieto Vesperinas 2002), cuyos involuntarios protagonistas fueron el pajarito bebedor y quien escribe estas líneas. ${ }^{2}$

En el artículo de César Sancho titulado «Faraday y la guitarra eléctrica» aparecen, nuevamente, conceptos básicos sobre las ondas y se presenta el fundamento físico para construir una guitarra eléctrica, que en esencia se basa en la ley de la inducción electromagnética. Así pues, ya tenemos (al menos) una excusa que justifique el estudio de esta parte del electromagnetismo

\footnotetext{
${ }^{2}$ La polémica del pajarito bebedor fue la que propició mi contacto con la Asociación de Profesores Amigos de la Ciencia - Eureka y, por ende, con esta revista. Por ello, deseo expresar mi agradecimiento al autor de la carta con los desafortunados comentarios sobre la enseñanza de la física (Lozano Leyva 2002).
} 
ante nuestros alumnos (amantes de la buena música): B. B. King, Eric Clapton, John Mayall, Gary Moore... no sonarían igual.

En «Microondas por chispa. Experimentos ópticos con microondas producidas con un equipo casero», Alejandro del Mazo Vivar explica cómo producir y detectar ondas electromagnéticas en la región de las microondas mediante el uso de aparatos sencillos. Además, el artículo contiene una documentada introducción histórica.

Enrique Vega Palas explica el sorprendente funcionamiento de algunos juguetes y artículos de regalo, los cuales se basan en la atracción o repulsión magnética y que parecen desafiar las leyes de la física, como sucede con la magia. De ahí proviene el título «Magia parece, magnetismo es».

Con un par de CD en desuso, un poco de hilo de cobre y un imán de neodimio, Celestí Capell Arqués explica en «Construcción de un altavoz con dos CD» el sencillo procedimiento para fabricar este dispositivo, omnipresente en nuestras vidas y en las de nuestros alumnos. De esta forma se pone de manifiesto (una vez más) la importancia, y ubicuidad, de la ley de inducción electromagnética.

La sección de experiencias de Física la cierra Jorge Barrio con el artículo «La complejidad de las mezclas sustractivas y su predicción a partir del análisis espectral». Como su título indica, este trabajo discute detalladamente las mezclas sustractivas de colores y nos alerta sobre los resultados paradójicos que podemos encontrar según sean los espectros de absorción o de transmitancia de las sustancias que se empleen. Según parece, no todo es del color del cristal con que se mire...

Las seis experiencias de Química comienzan con el trabajo «Azul, blanco, rojo. Homenaje a Lavoisier», escrito por Delia Ángeles García Liarte y Mateo Peña Martínez. La bandera de la República Francesa sirve de excusa para un entretenido juego de reacciones químicas en el cual va apareciendo de forma sucesiva los colores azul, blanco y rojo.

Con agua oxigenada, tan asiduamente empleada para desinfectar heridas, también se puede realizar espectaculares y divertidos experimentos de química, tal como nos muestran M. ${ }^{a}$ Luisa Aguilar Muñoz y Carlos Durán Torres en el trabajo titulado «Química recreativa con agua oxigenada».

Josep Corominas es capaz de organizar una fiesta con la química como protagonista, pues esta ciencia es la responsable de que los licores cambien de aspecto a voluntad del camarero o de que el anfitrión de la fiesta se coma una vela, todo ello ante la perplejidad del público. ${ }^{3}$ Para saber cómo se adquieren estas habilidades hay que leer el artículo «Fiesta química: licores que no lo son, bebidas que no hay que beber y alguna cosa de comer».

Miguel Ángel Gómez Crespo y Alfonsa Cañamero Lancha explican en «Juguetes y polímeros superabsorbentes» las posibilidades didácticas y lúdicas que ofrece el estudio de estos materiales, que se han convertido en compañeros habituales en nuestra vida cotidiana (pañales y compresas desechables, perlas de agua...).

La corrosión que sufren los metales en presencia de humedad es un fenómeno con importantes implicaciones tecnológicas. La vistosidad de las reacciones químicas involucradas en los procesos de corrosión queda de manifiesto en el trabajo «Experiencias sobre corrosión en metales de uso cotidiano», de Santiago Heredia Avalos.

\footnotetext{
${ }^{3}$ Siempre quise realizar esta experiencia, cuya descripción leí hace mucho tiempo en los magníficos libros de Tom Tit, donde aparece bajo la denominación de «Veilleuse comestible» (Tom Tit 1892) o «Le mangeur de chandelle» (Tom Tit 1906). Tuve el placer de presenciar cómo Josep Corominas se comía una vela en la edición de 2010 de El Pati de la Ciència (Corominas 2011).
} 
¿Y quién iba a pensar que las golosinas tienen cabida en el aula? Pues el trabajo de José Manuel Rodríguez Rodríguez y Juan A. Navarro de Tuero Bonán, titulado «Aprendiendo química con golosinas», dejará sin argumentos a los profesores que no permiten que sus alumnos lleven golosinas a clase, ya que éstos pueden replicar (educadamente) que lo hacen para aprender química.

El artículo «Microscopio de Leeuwenhoek», de Belén Garrido Garrido y Matilde Barcia González, inicia la sección dedicada a la Biología. Este trabajo, con referencias históricas al que fabricó Leeuwenhoek en el siglo xvII, explica la construcción de un sencillo microscopio, con el cual se puede observar células sanguíneas y de epidermis de vegetales.

Las propiedades terapéuticas atribuidas tradicionalmente al ajo quizá tengan relación con su actividad antimicrobiana. José Pedro López Pérez propone, en «Observación de la actividad antimicrobiana del ajo (Allium sativum) en el laboratorio de Educación Secundaria», una experiencia para realizar con los alumnos, la cual se basa en esta propiedad de esta conocida hortaliza.

El trabajo de Ana Paula L. Rodrigues titulado «De la geosfera a la biosfera: circulación de materia en la naturaleza» sirve para explicar a los alumnos cómo la lluvia ácida, tanto de origen antrópico como natural, afecta al desarrollo de la vida, especialmente, de los organismos más sensibles.

El trabajo anterior, a caballo entre la Biología y la Geología, da paso a «Lo que está bajo nuestros pies», un trabajo genuino de Geología, donde Paula C. de A. Maria Castelhano y Patrícia A. C. Fialho Azinhaga presentan unos sencillos modelos analógicos para ayudar a los estudiantes a entender las ondas sísmicas.

Concluye este monográfico con un par de artículos donde la Astronomía es la protagonista. En el primero, titulado «Sol, Tierra y Luna. Movimientos relativos y sus consecuencias», Sebastián Cardenete García recurre a los movimientos relativos entre los tres cuerpos celestes con los que estamos más familiarizados para explicar la sucesión día-noche, las estaciones, las fases de la Luna y los eclipses.

Esteban Esteban es el autor del segundo artículo dedicado a la Astronomía, que se titula «Recursos para visualizar algunas paradojas que se producen en el planeta Mercurio». En él se exponen y explican varias paradojas relacionadas con el movimiento del planeta Mercurio en torno al Sol.

Esperamos que los trabajos que contiene este número extraordinario de la REurEDC, así como la gran variedad de referencias bibliográficas, animen a los profesionales de la enseñanza a adentrarse en el apasionante mundo de la ciencia recreativa, capaz de proporcionar actividades formativas, vistosas y lúdicas. Al profesor corresponde ponerlas en contexto.

Los lectores juzgarán si ha valido la pena el esfuerzo (que ha sido enorme) que hemos realizado todos: autores, evaluadores, quien ha coordinado este monográfico y todo el equipo editorial de la Revista Eureka sobre Enseñanza y Divulgación de las Ciencias.

\section{Referencias}

Corominas J. (2011) Química a tot arreu, en Abril I., Garcia Molina R. El Pati de la Ciència, Universitat d'Alacant. http://rua.ua.es/dspace/handle/10045/15064

Lozano Leyva M. (2002) Física divertida. Sección de cartas al director en el diario El País. 29.04.02. http://www.elpais.com/articulo/opinion/Fisica/divertida/elpepiopi/200204 29elpepiopi_5/Tes 
Nieto Vesperinas M. (2002) Física divertida. Sección de cartas al director en el diario El País. 06.05.02.

Oliva J. M. (2008) Ciencia recreativa, educación científica en contextos y mediante recursos no formales, e investigación en didáctica de las ciencias. Revista Eureka sobre Enseñanza y Divulgación de las Ciencias 5 (1), 1. http://www.apac-eureka.org/revista/Volumen5/ Numero_5_1/Vol_5_Num_1.htm

Romero Peinado A. (2002) Sí, física divertida. Sección de cartas al director en el diario El País. 03.05.02. http://www.elpais.com/articulo/opinion/fisica/divertida/elpepiopi/200205 03elpepiopi_9/Tes

Solbes J., Lozano O., García Molina R. (2009) Análisis del uso de la ciencia recreativa en la enseñanza de materias científicas y técnicas en educación secundaria. Enseñanza de las Ciencias, Número Extra VIII Congreso Internacional sobre Investigación en Didáctica de las Ciencias, 1741-1745. http://ice.uab.cat/congresos2009/eprints/cd_congres/ propostes_htm/propostes/art-1754-1758.html

Tom Tit (1892) La Science Amusante (Deuxième Sèrie), p.187. París. Librairie Larousse.

Tom Tit (1906) Les Bons Jeudis, p.107. París. Vuibert et Nony Éditeurs. 